\section{Welche älteren AML-Patienten profitieren von einer allogenen Stammzelltransplantation?}

\begin{abstract}
Reduzierte Konditionierungsprotokolle ermöglichen auch älteren Patienten mit akuter myeloischer Leukämie (AML) eine allogene Stammzelltransplantation (allo-SZT). Etablierte Kriterien zur Einschätzung von Vor- und Nachteilen für den Einzelnen in dieser Altersgruppe fehlen bislang jedoch.
\end{abstract}

\begin{abstract}
n die retrospektive Studie gingen $\mathrm{Da}$ ten von 299 AML-Patienten ein, die zwischen 2003 und 2011 nach Cytarabin-basierter Chemotherapie eine Komplettremission (CR1) erreicht hatten. Die Patienten wurden in 2 Gruppen unterteilt. In Gruppe 1 (Alter: 60-65 Jahre; $\mathrm{n}=107)$ waren die Patienten ahängig von der Spenderverfügbarkeit entweder nach einer reduzierten Konditionierung (RIC) allogen transplantiert oder weiter chemotherapeutisch behandelt worden. Gruppe 2 (Alter: $\geq 66$ Jahre; $n=192$ ) galt altersbedingt als nicht für eine allo-SZT geeignet. Alle Patienten hatten ein ungünstiges oder intermediäres zytogenetisches Risiko.

Anhand der Daten der bis zu 65 Jahre alten Patienten wurden mithilfe der multivariaten Regressionsanalyse prä-
\end{abstract}

diktive Faktoren für den Nutzen einer allo-SZT ermittelt. Dabei war die alloSZT ein starker und unabhängiger positiver Prädiktor für das Gesamtüberleben (OS) und das rezidivfreie Überleben (RFS; beide $p<0,0001$ ). Ein ungünstiges zytogenetisches Risikoprofil und ein hoher HCT-CI (Hematopoietic Cell Transplant-Co-morbidity Index) waren negative prädiktive Faktoren. Das Gesamtüberleben nach CR1 betrug median 13 (1-54) Monate ohne und 31 (3-201) Monate mit allo-SZT.

Auch für die Patienten in Gruppe 2 wurden Einflussfaktoren für ein günstiges OS und PFS ermittelt. Ziel war es, diejenigen herauszufiltern, die doch noch von einer allo-SZT profitieren könnten. Als (negative) Prognosefaktoren erwiesen sich das Auftreten eines Rezidivs und ein schlechter Karnofsky-Performancestatus; beide waren mit einem signifikant schlechteren Überleben verbunden. Außerdem zeigte sich in der höchsten Altersgruppe der über 81-Jährigen das kürzeste rezidivfreie Überleben.

Höheres Alter ist bekanntermaßen der stärkste negative prognostische Faktor bei AML. Eine intensive Chemotherapie verlängert das Überleben älterer AMLPatienten, wie Studien ergaben, nicht. Unter einer allo-SZT mit RIC konnte dagegen in einer früheren Studie bei ausgewählten älteren Patienten eine Überlebensrate von rund $50 \%$ erreicht werden. Die Auswahl der für die allo-SZT geeigneten älteren Patienten spielt daher eine entscheidende Rolle.

Fazit: Die Ergebnisse dieser retrospektiven Studie könnten dazu beitragen, Auswahlkriterien für eine allogene Stammzelltransplantation bei älteren AML-Patienten zu ermitteln. Diese müssen jedoch in großen prospektiven Studien bestätigt werden.

Brigitte Schalhorn

Yamasaki S et al. Factors prognostic of eligibility for allogeneic HCT among older patients with AML-CR1 and adverse- or intermediate-risk cytogenetics. Ann Hematol. 2015;94(7):1159-65.

\title{
APL: Anthrazykline als Kombinationspartner von ATRA im Vergleich
}

Den Hauptpfeiler der Therapie einer akuten Promyelozytenleukämie (APL) bildet die Kombination aus All-Trans-Retinsäure (ATRA) und einer Anthrazyklin-basierten Chemotherapie. Nun wurde der Frage nachgegangen, ob sich Daunorubicin und Idarubicin als Kombinationspartner im Rahmen einer risikoadaptierten Therapie in Wirksamkeit und Verträglichkeit unterscheiden.

D ie Ergebnisse zweier Studien, eine Untersuchung der PETHEMA/HOVON-Gruppe und eine des internationalen APL-Konsortiums (IC-APL), boten Gelegenheit zu einem indirekten Vergleich. In der 1. wurde ATRA mit Idarubicin kombiniert, in der 2. mit Daunorubicin. In einer Matched-Pair-Analyse wurden 350 Patienten aus der PETHEMA/HOVON-Kohorte mit 175 der ICAPL-Studie verglichen. An beiden Studien hatten nur Patienten mit folgenden Charakteristika teilgenommen: Diagnose einer De-novo-APL mit t(15;17)-Translo- kation und/oder PML/RARA-Fusionsgen in den leukämischen Blasten, normale renale Funktion, keine kardiale Kontraindikation gegen Anthrazykline und ECOG-Performancestatus $<4$.

Mit der ATRA/Daunorubicin-Kombination erreichten die Patienten eine signifikant höhere Rate für komplette Remissionen als mit der Idarubicin-haltigen Kombination ( 94 vs. $85 \%, \mathrm{p}<0,002$ ). Die Ursachen für ein Induktionsversagen und die Zeitspanne bis zum Erreichen der kompletten Remission unterschieden sich nicht. Auch die kumulative Inzidenz der Rezidivraten sowie die krankheitsfreien Überlebensraten bei Patienten in kompletter Remission waren vergleichbar. Im IC-APL-Arm waren Gesamt- und ereignisfreies Überleben jedoch kürzer, hauptsächlich aufgrund höherer Letalität während Induktion und Konsolidierung.

Fazit: Die Kombination von Daunorubicin oder Idarubicin mit ATRA zeigt bei APL ähnliche antileukämische Wirksamkeit. Das ATRA/DaunorubicinSchema führte jedoch zu höheren Todesraten während Induktion und Konsolidierung.

Wolfgang Zimmermann

Sanz M A et al. All- trans retinoic acid with daunorubicin or idarubicin for risk-adapted treatment of acute promyelocytic leukaemia: A matched-pair analysis of the PETHEMA LPA2005 and IC-APL studies. Ann Hematol 2015; 94(8):1347-56. 